\title{
Photoelastic and numerical stress analysis of a 2D contact problem and 3D numerical solution for the case of a rigid body on a deformable one
}

\author{
A. Bilek \& F. Djeddi \\ Laboratoire d'Energétique, Mécanique et Matériaux, \\ Université Mouloud Mammeri, Tizi-Ouzou, Algérie
}

\begin{abstract}
A finite element analysis and a photoelastic stress analysis are conducted in order to determine the stress field developed in the pin on plan contact problem. Although this problem is relatively easy to study experimentally, the purpose here is to show the possibilities of the finite element method; after validation of the numerical procedure, problems with complicated geometry and boundary conditions can then be solved numerically. Isochromatic and isoclinic fringes, similar to the ones obtained experimentally by the photoelastic method, are obtained numerically over the whole model. The details of the finite element solution are fully given in the paper. Many studies have been achieved in order to separate the principal stresses and obtain their orientations (integration of the equilibrium equations ...) in order to compare them with the simulated results. However, this requires a high precision of measurement. Here, a whole field comparison of the experimental and numerical photoelastic fringes and a local analysis using the principal stresses difference, allowed us to validate the numerical approach. Relatively good agreements were obtained. A numerical solution for a three dimensional contact problem is also developed for a rigid parallelepiped on a deformable cylinder. The mesh was refined in the neighborhood of the contact zone in order to achieve better approximation of stresses. The loading is given by the limit conditions that are simply the imposed displacement. The calculated photoelastic fringes are obtained for various sections inside the model. These simulated fringes can be compared to the experimental ones which can be obtained by slicing the model and analyzing it in a plan polariscope. The program developed allows us to calculate stresses on any
\end{abstract}


given section inside the model, particularly in the neighborhood of the contact zone.

Keywords: birefringent, isoclinic, isochromatic, fringe, photoelasticity, contact, stress field, stress freezing, slicing.

\section{Introduction}

Several studies have shown that failure of mechanical parts occur generally in the neighborhood of the contact zones [1-4]. Stress initiation is mainly controlled by the shear stress mechanisms, particularly for metallic materials, by displacement of the dislocations on the crystallographic plans of higher densities. It is therefore very important to determine the type and the amplitude of the imposed mechanical solicitations.

Theoretical and numerical studies of the contact stresses are in some cases very complex. Several methods have been used to analyze this type of problem. In this work two methods have been used: the photoelasticity method and the finite elements method in order to determine stresses developed on the model.

The photoelastic fringes obtained experimentally with a plan polarized light are used to determine the values of the principal stresses difference over the whole model. To obtain the individual values of the stresses, that is to separate the principal stresses, several studies have been conducted by integrating the equilibrium equations (Zenina et al. [5] and Plouzennec [6]). However, a high precision is required in the unwrapping of the isochromatic and the isoclinic fringes obtained on the analyzer to determine respectively the difference and the direction of the principal stresses.

As already done in previous papers (Bilek et al. [7, 8]), it is sufficient to make a comparison between experimental and simulated fringes. Another comparison, which is more accurate, is made between experimental and simulated values of the principal stresses difference along the vertical axis of symmetry.

\section{Experimental analysis}

The model, made of epoxy resin (PLM-4R) mixed with a hardener, is mounted on a loading frame (figure 1) equipped with two dynamometers. The model is loaded via a steel pin of rectangular cross section $(12 \times 12 \mathrm{~mm})$, the load is set to $\mathrm{F}=1300 \mathrm{~N}$. The loading frame with the model is then positioned on the polariscope for analysis.

Plane polarized light is used to observe the photoelastic fringes. The isochromatic and the isoclinic fringes obtained on the analyzer are used to determine the values of the principal stresses difference and the principal stresses directions, particularly in the neighborhood of the contact zone.

The model in the shape of a parallelepiped $(67 \times 58 \times 10 \mathrm{~mm})$ is cut in the birefringent material. Poisson's ratio and Young's modulus, which are necessary to implement the numerical solution, are measured with the help of electrical strain gages mounted respectively on a tensile specimen and a cantilever beam. 


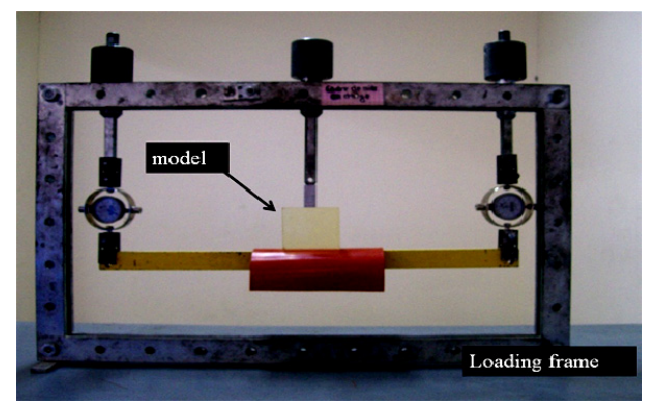

Figure 1: $\quad$ The model mounted on the loading frame.

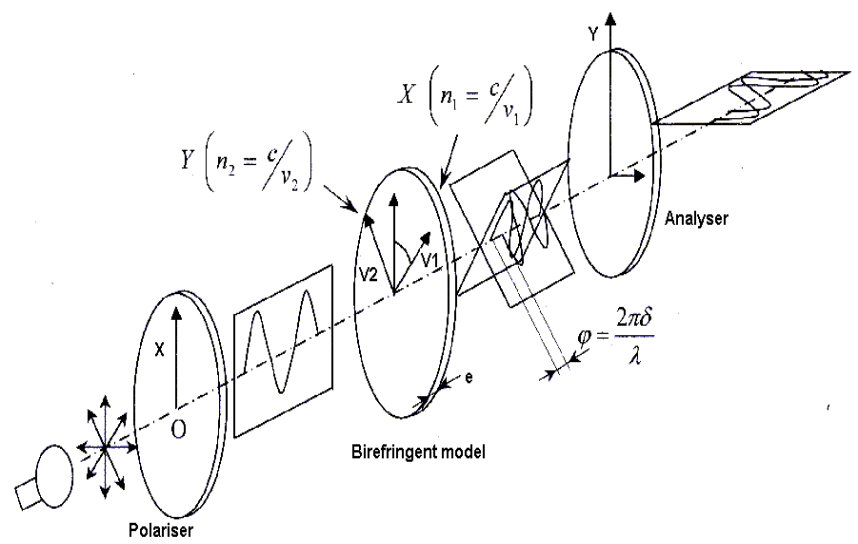

Figure 2: Light propagation through a photoelastic model.

Strains measured on the surface of the models allowed us to obtain easily these necessary values: $\mu=0.37$ and $E=2435 \mathrm{MPa}$.

Figure 2 shows the well known photoelastic method based on the birefringent phenomenon; the refractive index $n_{1}$ and $n_{2}$ which depend on stresses in the two principal directions induce a retardation angle $\varphi$. The light intensity obtained on the analyzer after traveling through the polarizer, the model and the analyzer has two terms: $\sin ^{2} 2 \alpha$ and $\sin ^{2} \varphi / 2$ which give respectively the isoclinic fringes and the isochromatic fringes (eq. (1)).

$$
\mathrm{I}=\mathrm{a}^{2} \sin ^{2} 2 \alpha \sin ^{2} \varphi / 2
$$

The isochromatic fringes allow us to obtain the values of the principal stresses difference on the model by using the well known relation (eq. (2)). This can only be done once the values of the fringe order $\mathrm{N}$ have been completely determined. The values of the fringe order $\mathrm{N}$ are determined either by the 
compensation technique or, whenever possible, by starting from a non stressed region on the model were $\mathrm{N}=0$. The fringe orders can then be easily deduced for the other fringes.

$$
\left(\sigma_{1}-\sigma_{2}\right) .=\mathrm{N}(\lambda / \mathrm{C}) / \mathrm{e}
$$

The ratio $\mathrm{f}=\lambda / \mathrm{C}$ called the fringe constant depends on the light wave used and the model material. Several solutions are available to obtain this value easily. Here, we subjected a beam $(40 \mathrm{~mm} \times 10 \mathrm{~mm}$ cross section) to a constant bending moment $(15000 \mathrm{~N} . \mathrm{mm})$ in a portion of its length (figure 3$)$, the light wave length used is $\lambda=546 \mathrm{~nm}$. We can see that the fringes are parallel to the horizontal axis of symmetry as one would expect; stresses at a same distance from the neutral axis are identical. We recall that isochromatics are loci of points having the same principal stresses difference. The isochromatic fringes are, therefore, parallel to the neutral axis of symmetry. Knowing the fringes orders and using the fact that the stress $\sigma_{2}$ is equal to zero (no load is applied in that direction), the value of the fringe constant can then be easily deduced by using (eq. (2)): $\mathrm{f}=11,65 \mathrm{~N} / \mathrm{mm} /$ fringe.

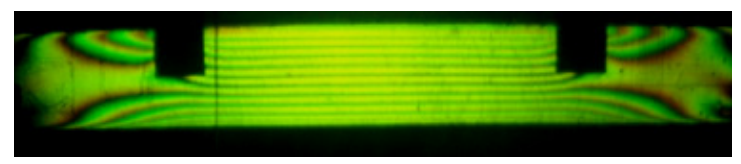

Figure 3: Model observed on a dark field polariscope.

Once the value of the fringe constant is determined, we can proceed to continue the analysis. The experimental isochromatic fringes (figure 4) on the analyzer of the polariscope are obtained with monochromatic light. Two quarter wave plates are added on the light path in order to eliminate optically the isoclinics that can hide the isochromatics which are necessary to determine the values of the principal stresses difference on the whole model, particularly in the neighborhood of the contact zone. These experimental isochromatic fringes will be compared to the simulated fringes obtained with the finite element analysis.

Another comparison is possible between the experimental isoclinics fringes and the simulated ones. The experimental isoclinics fringes (dark fringes on figure 5) are obtained for different positions of the polarizer and the analyzer. An image of the photoelastic fringes is recorded after each new position, the analyzer and the polarizer axes remain orthogonal to each other in order to always have a plan polarized light.

We recall that dark isoclinic fringes appear where the directions of the principal stresses coincide with the directions of the polarizer and the analyzer. The angle $\theta$ gives the position of the polarizer, the reference $\theta=0$ taken as the vertical axis. The isoclinic fringes can then be easily used to obtain the principal stress trajectories called also isostatics. Several numerical methods have been developed to obtain the isostatics rapidly and efficiently. 


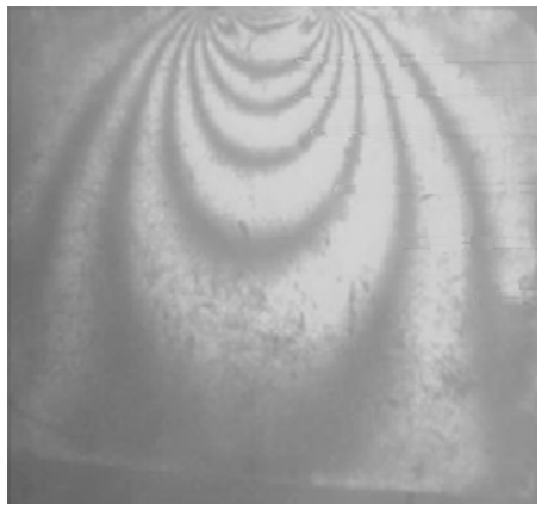

Figure 4: $\quad$ Isochromatic fringes obtained experimentally.

In this paper we are interested mainly in validating the finite element solution; it is therefore sufficient to compare the experimental and the calculated fringes. Another comparison, which is more accurate, is made between stresses obtained experimentally by analyzing the experimental isochromatic fringes and stresses obtained directly with the finites elements simulation, along the vertical axis of symmetry of the model.

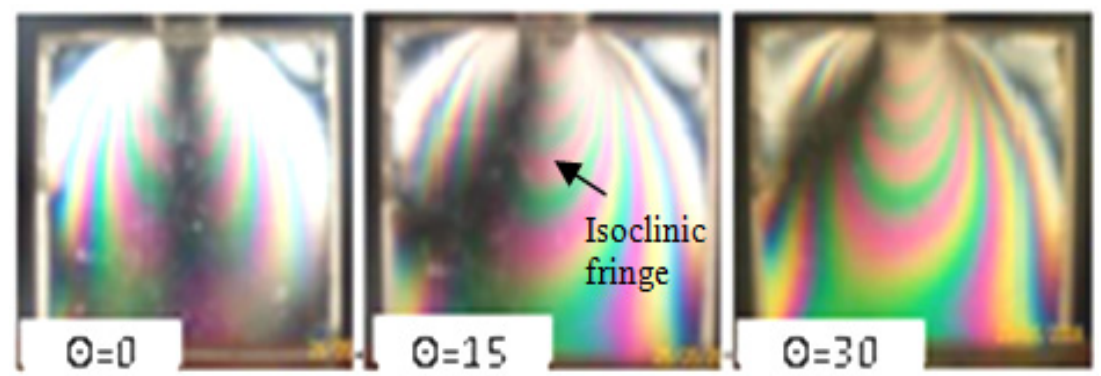

Figure 5: $\quad$ Isoclinic fringes at different angles.

\section{Numerical analysis}

In the finite element calculations, we considered that the material behaves everywhere as a purely elastic isotropic material. Young's modulus $\left(E_{1}=210000 \mathrm{MPa}, E_{2}=2435 \mathrm{MPa}\right)$ and Poisson's ratio $\left(\mu_{1}=0.3, \mu_{2}=0.37\right)$ for the two bodies in contact were introduced in the program. The mesh was refined in the neighborhood of the contact zone (figure 6) in order to achieve better simulation of stresses.

To achieve a better simulation of the applied load, an imposed displacement is applied to the model at the contact surface between the pin and the plan. The equivalent applied load is calculated then as the sum of the elementary vertical 


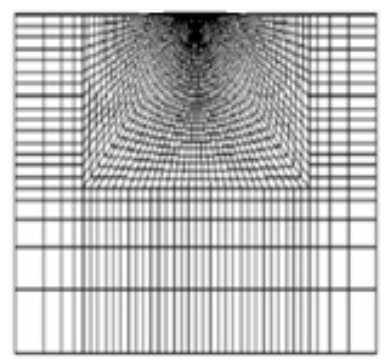

Figure 6: Finite element meshing.

load components at the nodes located at the lower surface of the model which is in contact with the loading frame.

Iterations on displacements at the contact nodes are stopped when the calculated corresponding load is equal to the value of the applied load within an acceptable error $(0.1 \%)$ set in the program. The isochromatic fringes represented by $\sin ^{2} \varphi / 2$ (eq. 1) are calculated then easily over the whole model. The details of the calculation are shown hereafter.

\subsection{Numerical calculation of the isochromatic fringes}

The following relation (eq. 3) which can be obtained readily from Mohr's circle for stresses allows us to calculate the principal stresses difference at any point of a stressed model.

$$
\left(\left(\sigma_{\mathrm{x}}-\sigma_{\mathrm{y}}\right)^{2}+4 \tau_{\mathrm{xy}}^{2}\right)^{0.5}=\sigma_{1}-\sigma_{2}=\mathrm{Nf} / \mathrm{e}
$$

The different values of the retardation angle $\varphi$ (eq. 4) can be calculated at any point on the model using the following relation:

$$
\varphi=2 \pi \mathrm{N}=\left(\left(\sigma_{\mathrm{x}}-\sigma_{\mathrm{y}}\right)^{2}+4 \tau_{\mathrm{xy}}^{2}\right)^{0.5} 2 \pi \mathrm{e} / \mathrm{f}
$$

The different values of $\sin ^{2} \varphi / 2$ which represents the isochromatic fringes (figure 7 , left) can then be easily calculated over the whole model. A comparison can then be made with the isochromatic fringes obtained experimentally (figure 7 , right). We can see relatively good agreement; however in the neighborhood of the contact zone we can see some discrepancies.

Figure 8 shows the variation of the principal stresses difference along the vertical axis. The value increases to approximately $6 \mathrm{MPa}$ and then decreases away from the contact zone. For the experimental solution it is difficult to determine the stresses close to the contact zone.

The following hypotheses can be considered to explain these differences. For the finite elements procedure, the constitutive equations (mechanical behavior) taken for the material, the mesh sizes especially close to the contact zone and the boundary condition are not exactly the same as the real ones. Also, for the experimental procedure the loading is not perfectly symmetrical and the spatial resolution in the neighborhood of the contact zone is limited. 

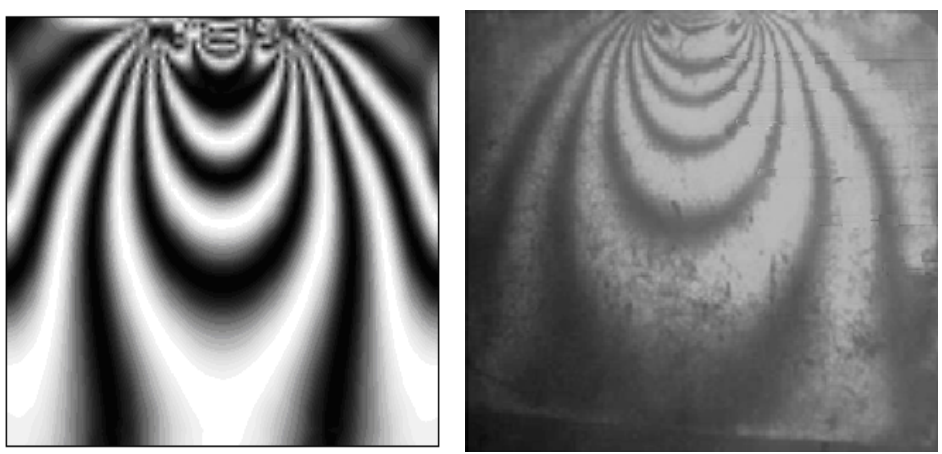

Figure 7: $\quad$ Simulated isochromatic fringes (left), experimental ones (right).

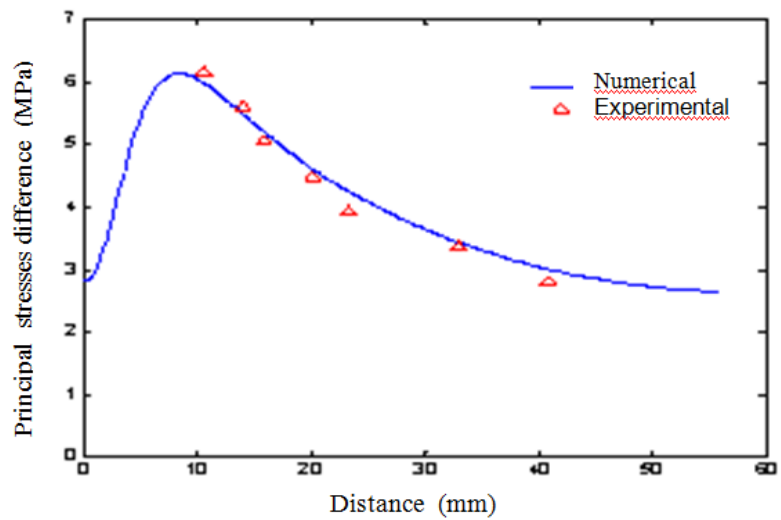

Figure 8: $\quad$ Principal stresses difference along the vertical axis.

The term $\sin ^{2} 2 \alpha$ represents the isoclinic fringes which are loci of points where the principal stresses directions are parallel to the polarizer and the analyzer. In the simulation program, the different values of the isoclinic parameter $\alpha$ can be calculated with the following relation (eq. 5) which can be obtained readily from Mohr's circle for stresses:

$$
\alpha=\operatorname{arct}\left(2 \tau_{\mathrm{xy}} /\left(\sigma_{\mathrm{x}}-\sigma_{\mathrm{y}}\right)\right)
$$

The program calculates the different values of the parameter $\alpha$. The image corresponding to the isoclinic fringes $\left(\sin ^{2} 2 \alpha\right)$ can then be calculated and displayed (figure 9). The comparison is then possible with the experimental isoclinic fringes which are the dark fringes obtained experimentally (figure 5). Experimentally it is not possible, of course, to observe the isoclinics alone whereas for the finite element solution this is possible. Good agreement was obtained between the experimental and the simulated isoclinic fringes. 


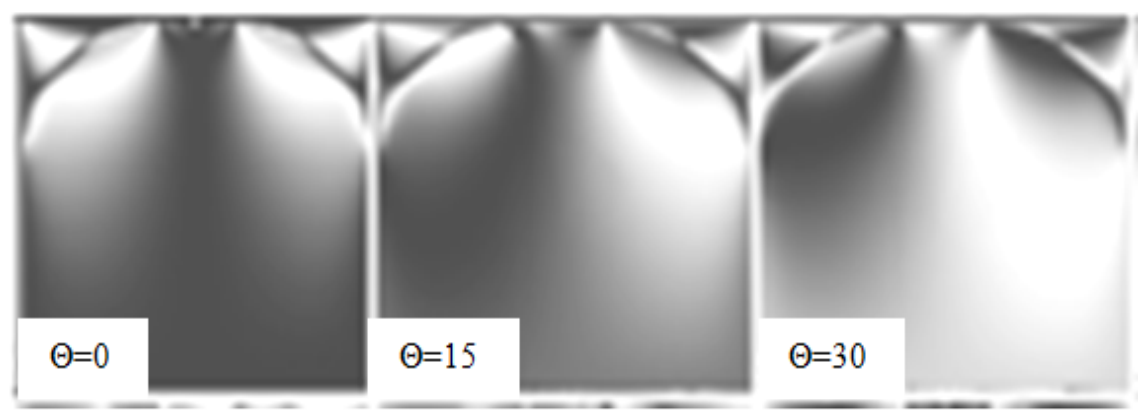

Figure 9: $\quad$ Simulated isoclinic fringes.

\section{Numerical solution for the case of a rigid parallelepiped on a deformable cylinder}

The birefringent cylindrical specimen (35mm diameter and $50 \mathrm{~mm}$ length) is subjected to a compressive simulated load by a steel rigid parallelepiped (cross section 10x10 mm). The purpose here is to calculate stresses on any chosen plan perpendicular to the longitudinal axis of the cylinder (figure 10), particularly in the neighborhood of the contact zone. Simulated fringes similar to the photoelastic fringes obtained experimentally can then be calculated and displayed.

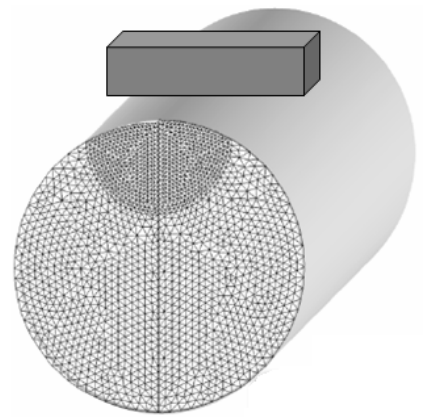

Figure 10: Experimental model.

The same as for the previous two dimensional case, displacements are imposed on the upper surface of the model at the nodes that will come into contact after the load is actually applied. The imposed displacement is calculated separately at each node; the imposed displacement decreases from a set value for the first point of contact and decreases as we move away from this point. The corresponding applied load is then calculated as the sum of the elementary vertical loads on the nodes at the lower surface of the model which is in contact with the loading frame. 


\subsection{Simulation for the case of mechanical slicing}

If one uses the stress frizzing experimental technique to analyze the stress field by mechanically slicing the model and then analyzing stresses on a regular polariscope with circularly polarized light (quarter wave plate are added to the polariscope in the light path) to obtain the photoelastic fringes, the light intensity on the analyzer is given in by the following relation (eq. (6)):

$$
\mathrm{I}=\mathrm{a}^{2} \sin ^{2}(\varphi / 2)
$$

In order to analyze stresses in three dimensional models we generally use the stress freezing technique which consists on locking stresses in the model. The model is then mechanically sliced with chosen plans in order to allow a two dimensional analysis on a regular polariscope.

Since for the stress freezing technique stresses should be locked inside the model at the stress freezing temperature, fringe constant and Young's modulus of the model material should be determined at this temperature. These characteristics, $\mathrm{f}=0.44 \mathrm{~N} / \mathrm{mm} /$ fringe and $\mathrm{E}=15.9 \mathrm{MPa}$, are taken from reference (Bilek et al. [4]). These values should then be introduced in the finite elements program in order to implement the solution.
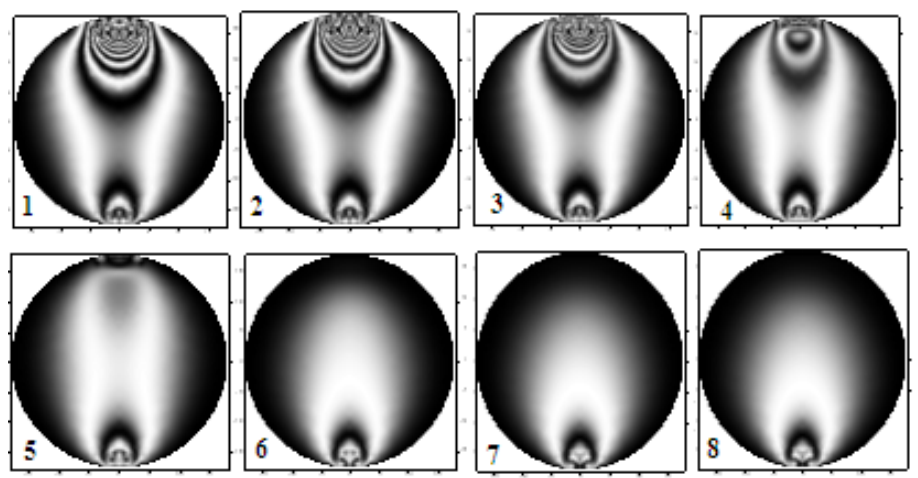

Figure 11: Simulated isochromatic fringes.

In the finite element solution, it is necessary to select the thickness of the slice to be isolated. The slice thickness should be small enough so that stresses remain relatively constant across the thickness. Here, we choose $10 \mathrm{~mm}$ which corresponds approximately to the generally used thickness for a two dimensional model. This process is repeated along the length of the cylinder in order to determine the variation of stresses in the whole volume. Figure 11 shows the simulated isochromatic fringes obtained with software package castem. 


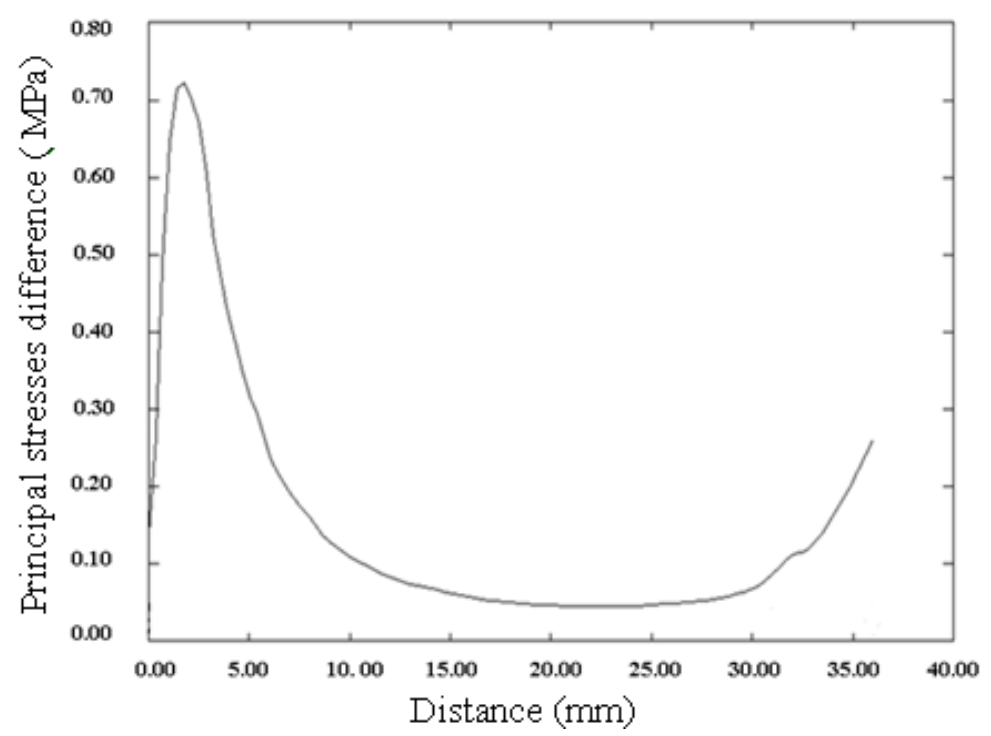

Figure 12: Principal stresses difference along the vertical axis of symmetry.

For a same slice (image 1, figure 11), we can see that stresses decrease as we move away from the contact zone. Also as we move along the cylinder (image 1 through 8) we see less isochromatic fringes which show clearly that stresses decrease. The stresses at the lower of the cylinder remain relatively constant; the load is uniformly distributed over the surface of contact with the loading frame.

The principal stresses difference along the vertical axis of symmetry increases to a maximum value of about $0.72 \mathrm{MPa}$ and then decreases as we move away from the contact zone. The value of the principal stresses difference increases again, as we move close to the contact zone of the cylinder with the loading frame, to reach a value of $0.27 \mathrm{MPa}$. This graph can be obtained along the vertical axis for any plan along the length of the cylinder.

\section{Conclusion}

We have shown through the study of a two dimensional model that the simulation of stresses developed on a plan loaded with a pin gives relatively good agreements with the experimental ones. The isochromatic and the isoclinic fringes are comparable to the photoelastic fringes obtained on a regular polariscope. A solution for a three dimensional problem is developed. The isochromatic fringes are obtained for various sections along the cylinder. The principal stresses difference can be easily calculated in the volume of the cylinder. This allows us to locate the zones of stress concentration which is of great importance in the design of mechanical components. An experimental solution either by the stress freezing method or the optical slicing method can be used for comparison purposes. 


\section{References}

[1] Mihailidis, A., Bakolas, V., \& Drivakovs, N., Subsurface stress field of a dry line Contact. Wear V. 249, pp 546-556, 2001.

[2] Burguete, R. L. \& Patterson, E. A., A photoelastic study of contact between a cylinder and a half-space. Experimental Mechanics V.7, No. 3, 1997.

[3] Kogut, L. \& Etsion I., Elastic-Plastic contact analysis of a sphere and a rigid flat. Journal of Applied Mechanics, V.69, pp.657- 662, 2002.

[4] Bilek, A., Dupré, J. C., Ouibrahim, A. \& Bremand, F., 3D Photoelasticity and numerical analysis of a cylinder/half-space contact problem, Computer methods and experimental measurements for surface effects and contact mechanics VII, pp 173 -182, 2000.

[5] Zenina, A., Dupré, J.C. \& Lagarde, A., Separation of isochromatic and isoclinic patterns of a slice optically isolated in a 3-D photoelastic medium. Eur. J. Mech. A/Solids 18, pp. 633-640, 1999.

[6] Plouzennec, N., Développement de Processus d'analyse d'Images en Photoélasticimétrie par un feuillet plan obtenue par découpage mécanique et optique. Thèse de l'Université de Poitiers, 1996.

[7] Bilek, A., Dupré, J. C., Brémand, F. \& Ouibrahim, A., Studies of contact problems by $3 \mathrm{D}$ photoelasticity, comparison with finite element analysis, International conference on experimental mechanics, Italy, 2004.

[8] Bilek, A., Ouibrahim, A., Brémand, F. \& Dupré, J. C., Experimental and numerical analysis of a cylinder/cylinder contact problem. ETDCM8, Experimental techniques and design in composite materials, Italy 2007. 